\title{
Aspects of Adopting CIM Standard by ENTSO-E and Common Grid Model Exchange Standard Implemention in Transelectrica
}

\author{
Oana Mânicuță \\ Power Engineering Faculty, University "Politehnica" of Bucharest, Bucharest 060042, Romania
}

Received: October 28, 2015 / Accepted: November 17, 2015 / Published: December 31, 2015.

\begin{abstract}
This paper presents theoretical issues about CIM (common information model) and CGMES (common grid model exchange standard), which will allow the exchange of data bases for power flow, short circuit and dynamics at ENTSO-E (European network for transport system operators for electricity) level. Grid model exchange is a complex process covering a variety of use cases, which include the exchange of equipment information, topology information, information on power system state variables, steady state hypothesis information. CGMES is an ENTSO-E standard that follows the CIM guidelines of IEC (International Electrotechnical Commission) TC57/WG13 in order to comply with the requirements of the European network codes. There are mentioned in the paper the challenges of implementing CIM and CGMES in Transelectrica for the planning cases.
\end{abstract}

Key words: CGMES, CIM standard, IEC 61970.

\section{Introduction}

In ENTSO-E (European network for transport system operators for electricity), the network development databases exchanges such as planning scenarios or day ahead congestion forecast need to be done in a common format that has to be followed by all TSOs (transport and system operators). This common format, named the "CIM (common information model)", will allow the exchange of data bases for power flow, short circuit and dynamics at ENTSO-E level.

CGMES (common grid model exchange standard) is an ENTSO-E standard that follows the CIM guidelines of IEC (International Electrotechnical Commission) TC57/WG13 in order to comply with the requirements of the European network codes.

Corresponding author: Oana Mânicuță, Ph.D. student, research fields: renewable energy, CIM and CGMES format, dynamic simulations.

\section{Aspects about the Common Information Model and Common Grid Model Exchange Standard Modeling}

There are two official versions of the CIM common format: CIM 14 (released in November 2009-for power flow data exchanges) and CGMES or CIM 16 (released in August 2014-for power flow, short circuit and dynamic data exchanges).

In order to illustrate changes made within their territory, each TSO is viewed as a single authority (TSO set based on the MAS - model authority sets) that has to provide the following files: an EQ (equipment file), a TP (topology file), a SV (state variables file) and a DY (dynamic data file). These files correspond to the first version of the CIM/XML (common information model/extensible markup language) profile, which was released in November 2009 [1].

The CIM format is described by a suit of standards of the IEC, IEC 61970, which define the need for an 
application (API - application program interface) for an EMS (energy management system). CIM is described by means of the UML (unified modeling language), a modelling language often used for visualization a system's structure in an object-oriented manner. In order for it to be used by software programs, CIM must use schemas that contain object characteristics and the relationships between these objects. The CIM format was created with enterprise architect [2].

The NERC (North American Electric Reliability Council), DEWG (data exchange working group), CPSM (common power system modeling group) worked to create the IEC 61970-452 CIM model exchange standard, which is used to describe data needed for development studies and the state estimator.

In Fig. 1 [1], it is described the relationships between the profile groups in IEC 61970 .

The IEC 61970 standards contain the following parts (Fig. 1):

- Part 452: "CIM model exchange specification/equipment model" - it describes the sets of classes, class attributes ant the relationships between them for the state estimator and power flow applications;

- Part 456: "solved power system state interface/state variables \& topology"-used to describe the exchange of power flow results;

- Part 552: CIM/XML model exchange format;

- Part 453: "diagram layout profile"-schematic display layout exchange profile group.

The "Reference Manual for Exchanging Standard Power System Dynamic Models: Based on the IEC 61970 Common Information Model" from the EPRI (Electric Power Research Institute) CIM for dynamic models project describes the main dynamic models used by General Electric, Siemens Power Technologies International PTI, DigSilent and Tractebel/RTE (Réseau de transport d'électricité) [3].

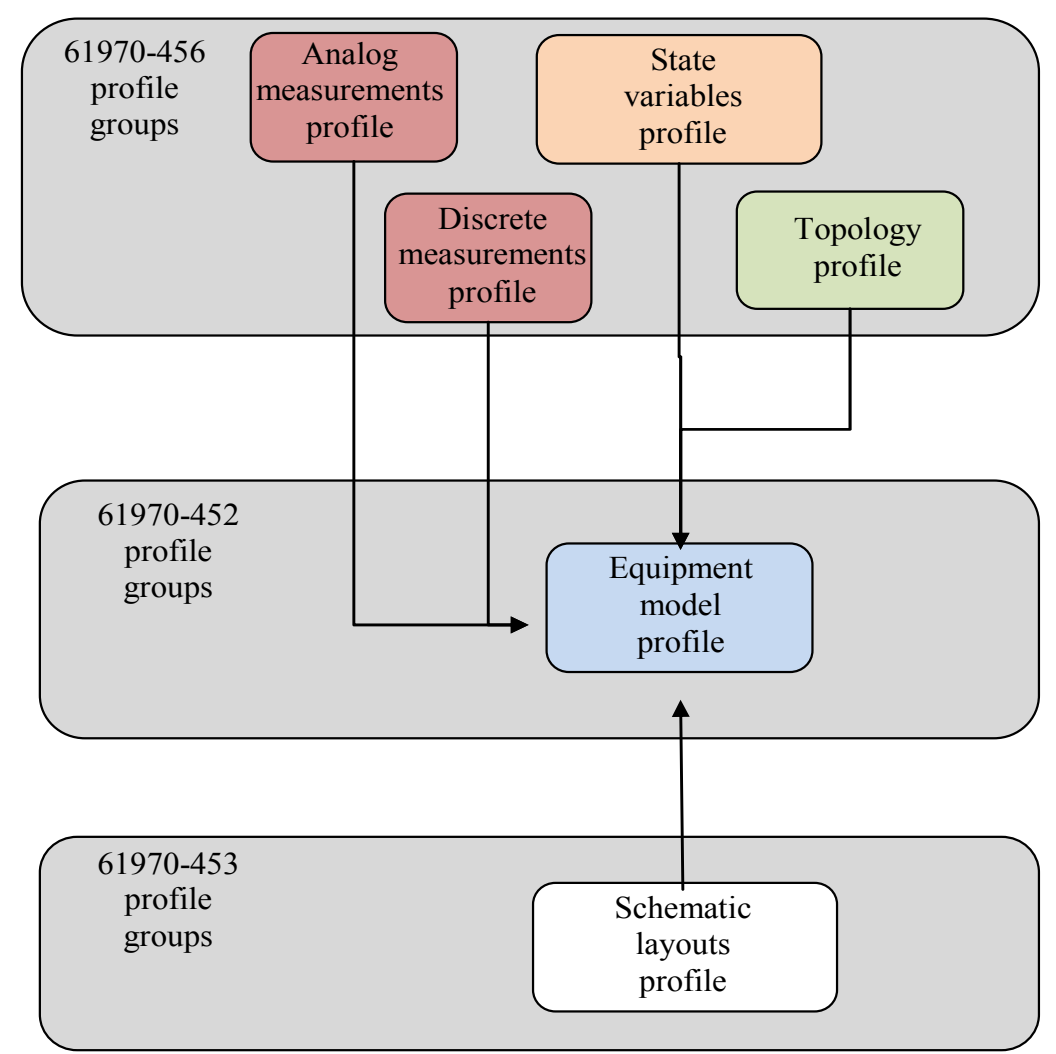

Fig. 1 Relationship between IEC 61970 standards. 


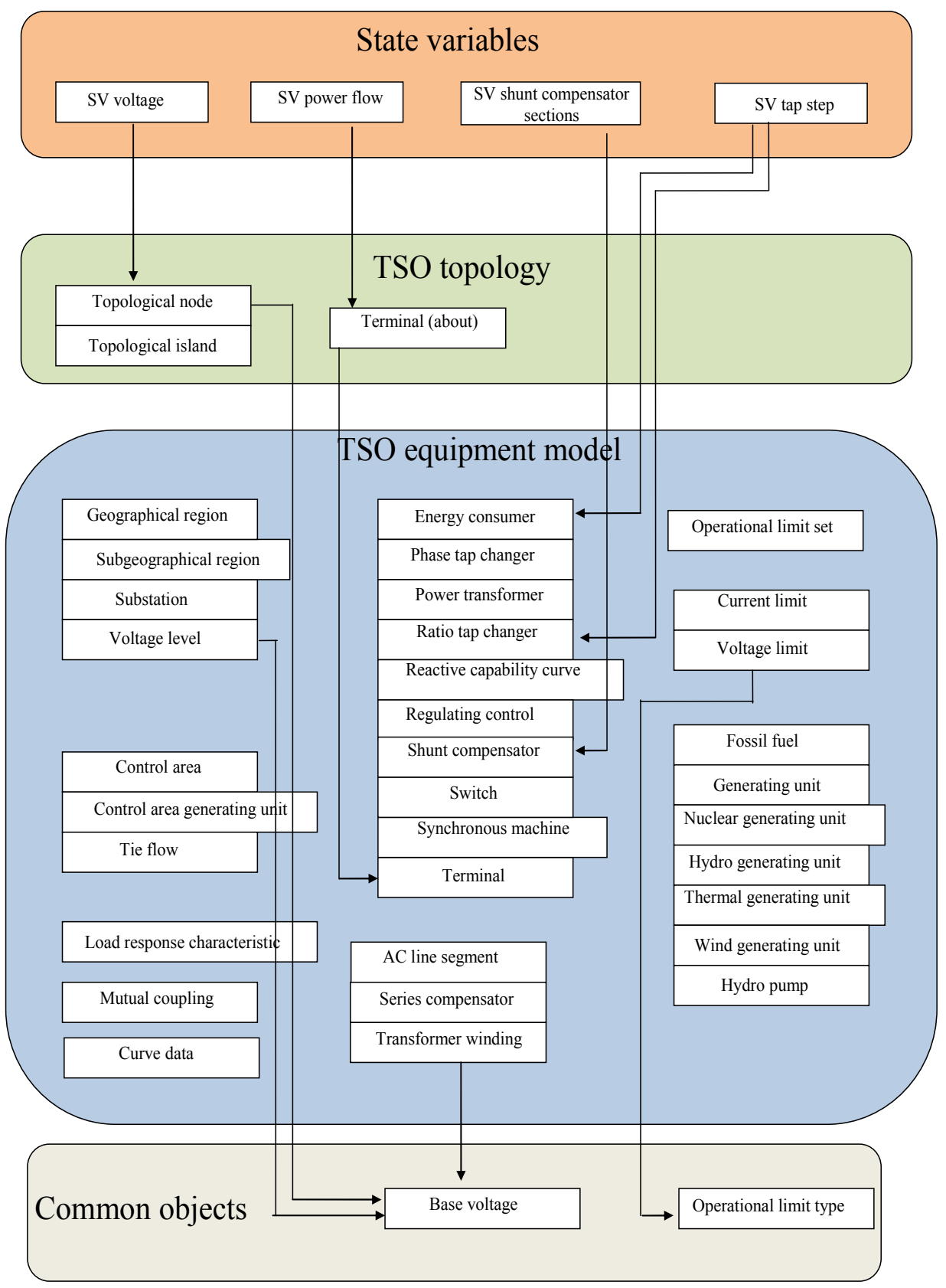

Fig. 2 The main classes in EQ, TP and SV files.

The main classes are described in Fig. 2 [1].

The second edition of the profile, called CGMES (common grid model exchange standard), contains several improvements and two new files: DI-diagram layout file and GL-geographical location, a GIS - geographic information system data file [4]. CGMES is based on CIM UML 16, version 25 (Fig. 3) [5].

The CGMES format (version 2.4.15) was released in August 2014 and is based on the IEC standards in place for UML 16, version 25 [4]:

- IEC 61970-552: CIM XML model exchange format;

- IEC 61970-301: CIM base;

- IEC 61970-302: CIM for dynamics specification;

- IEC 61970-452: CIM static transmission network model profiles;

- IEC 61970-453: diagram layout profile; 
- IEC 61970-456: solved power system state profiles;

- IEC 61970-457: CIM for dynamics profile;

- IEC 61968-4: application integration at electric utilities - system interfaces for distribution management_-Part 4: interfaces for records and asset management.

The EQ file contains information regarding the TSO's equipment. At the equipment level, there are no connections to the boundary set file.

The TP file contains information regarding electrical connectivity.

The SV file contains all power flow data.

The DY file contains data necessary for dynamic analysis.
The DL (diagram layout) file contains the physical representation of the model.

The GL (geographical layout) file contains the GIS coordinates of the equipment.

CIMdesk (CIM-based data engineering solution kit) is a program that offers the possibility of importing, exporting, validating, editing and visualizing data bases in CIM and CGMES formats (Fig. 4) [6].

Data exchange in CIM/CGMES format can be achieved by means of the following programs: Aktiengesellschaft für Produkte und Systeme der Informationstechnologie (PSI AG)-PSI control, General Electric-energy management service (enterprise gateway), DIGSilent $\mathrm{GmBH}$ (power factory), Alstom Grid (e-terrasource/e-terraplatform),

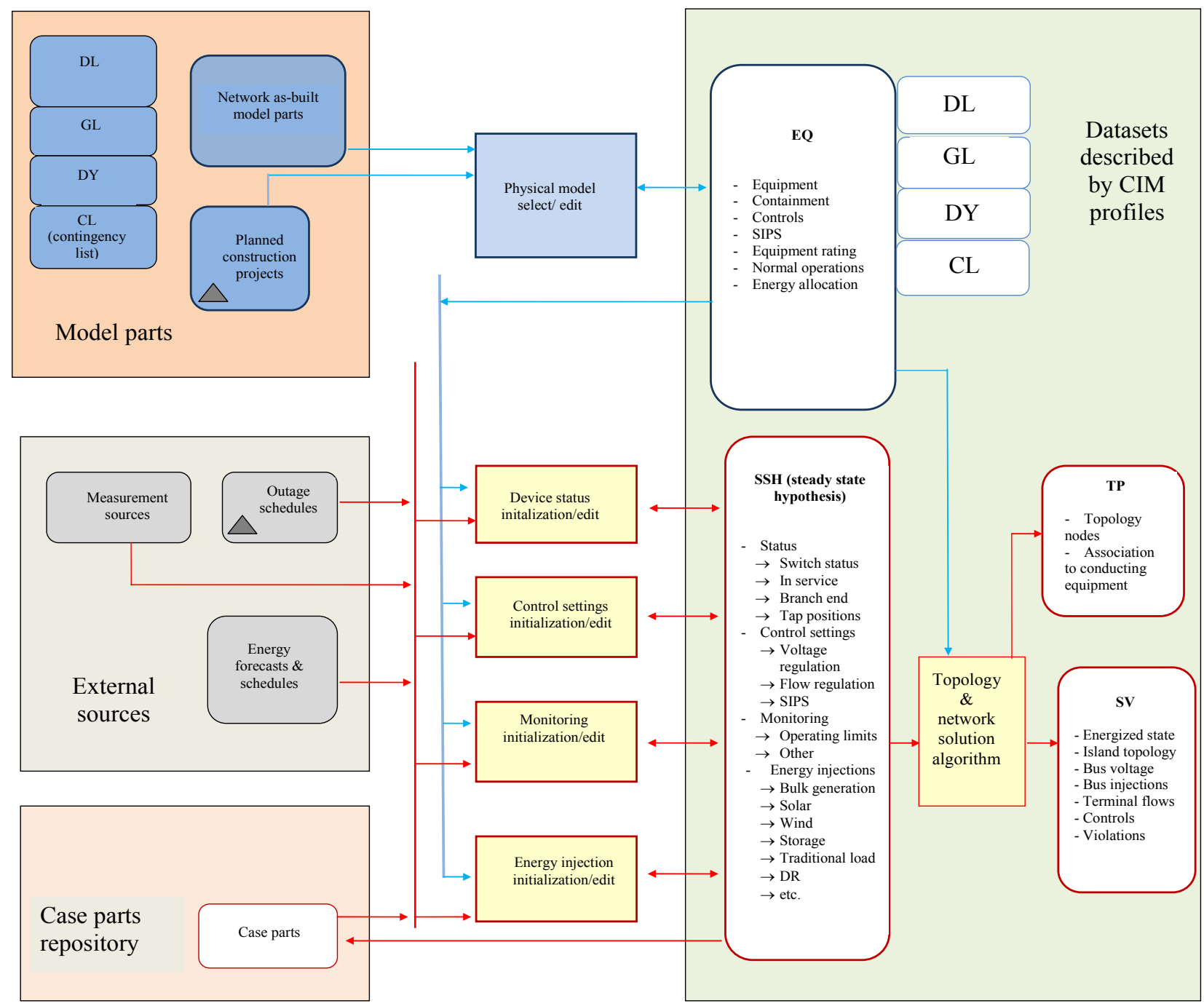

Fig. 3 The second edition of the CIM format (CGMES). 


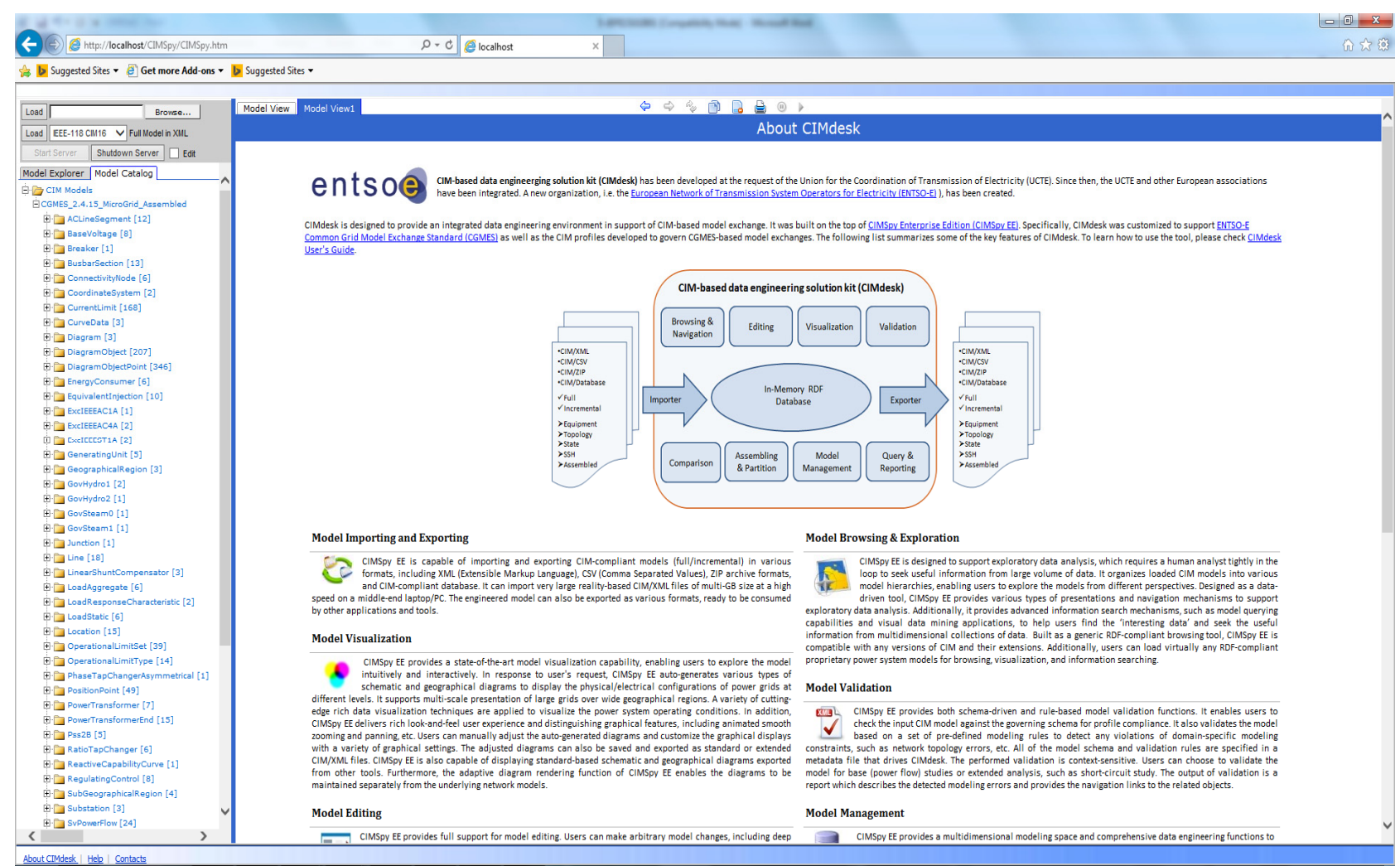

Fig. 4 CIMDesk interface.

RTE (convergence), Centro Elettrotecnico Sperimentale Italiano S.P.A (Spira), Schneider Electric DMS (distribution management system) — transmission network analyzer, Unicorn Systems A.S. (common tool for data exchange and security assessment-CTDS), Siemens AG (power system simulator/SINCAL) and PSS/ODMS (power system simulator/operational data management simulator) [7].

The CGMES can also be used for modelling wind farms and HVDC (high voltage direct current), both for power flow studies and dynamics.

The document that establishes the guidelines for modelling wind farms is named IEC 61400-27-1 "electrical simulation models — wind turbines" and has been developed by IEC WG 27.

CIM format is used successfully in America [8, 9].

\section{CIM and CGMES Implementation in Transelectrica}

Transelectrica (the Romanian Transport System
Operator) is using PSS/ODMS for exchanging the models in CIM and CGMES format. PSS/ODMS single user edition developed by Siemens PTI USA represents a restricted version of PSS/ODMS enterprise edition. Due to the fact that, PSS/ODMS requires data base handling, the application uses Microsoft SQL (structured query language) Server 2008 express edition or ORACLE (Oak Ridge automatic computer and logical engine). PSS/ODMS enterprise edition is used for data bases that are changed in real time, such as SCADA (supervisory control and data acquisition) systems [10].

The challenges of implementing CIM and CGMES for planning cases in Transelectrica were:

- to ensure that, the rdf:IDs (unique identifiers of elements of the model) are globally unique to the other ENTSO-E models;

- exchanging the fuel types for the generators (nuclear, thermal, hydro, wind, solar, biomass, etc.);

- the different way of modelling of the transformers between different planning tools; 
- consolidating the nodes into substations (a substation is described in Ref. [1] as a collection of equipment for purposes other than generation or utilization, through which electric energy in bulk is passed for the purposes of switching or modifying its characteristics).

\section{Conclusions}

This paper presented theoretical issues about common information model and common grid model exchange standard, which will allow the exchange of data bases for power flow, short circuit and dynamics at ENTSO-E level.

The CIM for grid model exchange enables exchanges for the data necessary for regional or pan-European grid development studies, and for future processes related to network codes.

Grid model exchange is a complex process covering a variety of use cases, which include the exchange of:

- equipment information, which contains power system equipment data;

- topology information, which contains topology related information for the grid elements;

- information on power system state variables, which contains the results from initial load flow simulation of the system;

- steady state hypothesis information, which is valid for newer standards and provides information on load and generation values as well as other input parameters necessary to perform load flow simulations.

In addition, grid model exchange can benefit from information related to dynamics, diagram layout and geographical location for elements in the power system.

\section{Acknowledgments}

The work has been funded by the SOPHRD (sectoral operational programme human resources development) 2007-2013 of the Ministry of European Funds through the Financial Agreement no. SOPHRD/159/1.5/S/132397.

\section{References}

[1] ENTSO-E. 2009. "Common Information Model (CIM) - Model Exchange Profile.” ENTSO-E.

[2] Ivanov, C. 2013. "One Edition with a Theme on CIM." Presented at the CIM User Group Meeting, Ljubljana, Slovenia.

[3] EPRI (Electric Power Research Institute). 2009. "Reference Manual for Exchanging Standard Power System Dynamic Models-Based on the IEC 61970 Common Information Model (CIM).” EPRI.

[4] ENTSO-E. 2014. "Common Grid Model Exchange Standard (CGMES)-Version 2.4-Based on IEC Common Information Model.” ENTSO-E.

[5] Lars-Ola, O. 2015. "TC57-Review of IEC 61970-456." Presented at the CIM User Group Meeting, Gdansk, Poland.

[6] Power Info LLC. 2015. "CIM Data Engineering Solution Kit (CIMdesk)." Presented at the ENTSO-E, Brussels, Belgium.

[7] ENTSO-E. 2015. "Conformity Registry.” ENTSO-E.

[8] Haq, E. 2007. "CIM-Based Network Models in a Multi-vendor Environment at CAISO." Presented at the IEEE PES General Meeting, Tampa, Florida.

[9] Haq, E., Haller, D., Rahman, K. A., and Iverson, B. 2011. "Use of Common Information Model (CIM) in Electricity Market at California ISO." IEEE.

[10] SIEMENS. 2014. "ODMS Product Overview." SIEMENS 